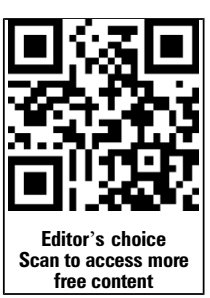

free content
${ }^{1}$ Clinica Ortopedica e Traumatologica II-Lab. di Biomeccanica, ed Innovazione Tecnologica, Istituto Ortopedico Rizzoli, Bologna, Italy ${ }^{2}$ Isokinetic FIFA Medical Center of Excellence, Bologna, Italy

\section{Correspondence to} Dr Alberto Grassi, Clinica Ortopedica e Traumatologica II - Lab. di Biomeccanica, ed Innovazione Tecnologica, Istituto Ortopedico Rizzoli, Via di Barbiano, 1/10, Bologna 40136, Italy;

alberto.grassi3@studio.unibo.it

Accepted 18 May 2015 Published Online First 10 June 2015

\section{CrossMark}

To cite: Grassi A, Zaffagnini $S$, Marcheggiani Muccioli GM, et al. Br J Sports Med 2015;49: 1295-1304.

\title{
After revision anterior cruciate ligament reconstruction, who returns to sport? A systematic review and meta-analysis
}

\author{
Alberto Grassi, ${ }^{1}$ Stefano Zaffagnini, ${ }^{1}$ Giulio Maria Marcheggiani Muccioli, ${ }^{1}$ \\ Maria Pia Neri, ${ }^{1}$ Stefano Della Villa, ${ }^{2}$ Maurilio Marcacci ${ }^{1}$
}

\begin{abstract}
Background Return to sport and to pre-injury level represents an important outcome after both primary and revision anterior cruciate ligament $(A C L)$ reconstructions. Purpose The aim of the present meta-analysis was to determine the return to sport rate after revision $A C L$ reconstruction.

Material and methods A systematic search was performed of the MEDLINE, Embase and the Cochrane Central Register of Controlled Trials Databases. All the studies that reported return to sport, return to pre-injury sport level and return to high level/competitive sport was considered for the meta-analysis. The overall pooled mean of post-operative knee laxity and pooled rate of positive pivot-shift and objective International Knee Documentation Committee (IKDC) categories was calculated as well.
\end{abstract}

Results Overall, 472 abstracts were identified and screened for inclusion and only 16 studies reported the rate of return to any level of sport activity at the final follow-up of 4.7 years (range 1.0-13.2 years), showing a pooled rate of $85.3 \%(\mathrm{Cl} 79.7$ to 90.2$)$. The return to pre-injury sport level was achieved in $53.4 \%(\mathrm{Cl} 37.8$ to 68.7) of cases. Normal or quasi-normal objective IKDC, less than $5 \mathrm{~mm}$ of side-to-side difference at arthrometric evaluations and grade I-II pivot-shift test were reported in $84 \%, 88 \%$ and $93 \%$ patients, respectively.

Conclusions In spite of almost 8 patients out of 10 returning to sport after revision $A C L$ reconstruction and showing good stability, only half of the patients returned to the same pre-injury sport level.

\section{INTRODUCTION}

Return to sport and especially to pre-injury levels represents for patients one of the most important clinical outcomes after ACL reconstruction. Despite clinical satisfaction and adequate knee range of motion (ROM), stability and muscle strength, the ability to perform sports activity is believed to represent a good indicator of overall knee health and of the ability of the joint to sustain external load and stress, particularly in high-level, competitive and pivoting activities. ${ }^{1}$ However, in certain circumstances, the normalised objective knee status and recovered muscle strength do not correspond to the ability to perform sport, especially when psychological recovery is not reached. ${ }^{2-5}$

With regard to this, the outcomes of return to sport after primary ACL reconstruction have been extensively investigated through high-level clinical studies and well-conducted meta-analyses. ${ }^{1} \quad 6 \quad 7$ In contrast, the outcomes of revision ACL reconstruction derive mostly from level IV retrospective studies. ${ }^{8}$ A recent meta-analysis of 21 studies investigated the main clinical outcomes of revision ACL reconstruction, reporting a return to unrestricted activities or to the previous levels of activity in 54\%, but without clearly defining a precise return to sport activity definition. ${ }^{8}$ Moreover, since then, clinical studies reporting the results after revision ACL have almost doubled, underlining the increasing interest in this surgical procedure. If the increased number of ACL lesions, estimated at around 36.9/100 000 person-year, ${ }^{9}$ and the failure rate of primary ACL reconstruction of $7-10 \%{ }^{10}$ are considered, it is not difficult to understand the burden of the revision ACL reconstruction procedure and its importance in the field of sports medicine and traumatology.

Nowadays ACL revision surgery, once considered but a salvage procedure, is increasingly obtaining results which are more and more similar to primary reconstruction mainly regarding clinical outcomes and knee stability, ${ }^{8}{ }^{11}$ owing to technical surgical advances. However, the ability to perform sports activity after revision ACL reconstruction remains a not adequately investigated issue, as single studies reported the return to pre-injury sport level ranging from $0 \%$ to $100 \% .^{12} 13$

The primary aim of the present meta-analysis was to synthesise the current evidence related to sports activity after revision ACL reconstruction, and to determine the overall average postoperative return-to-sports and return to pre-injury sport level rates. The secondary aim was to summarise key objective knee function measures, including the International Knee Documentation Committee (IKDC) score, the anteroposterior knee laxity and pivot shift test.

\section{MATERIAL AND METHODS \\ Study design}

A meta-analysis was performed in accordance with the PRISMA Statement for Reporting Systematic Reviews and Meta-Analyses. ${ }^{14}$ No protocol exists for this study, which was exempt from review by Ethical Committee approval for only those studies that involved human participants, as no patients were enrolled in the study and nor were medical or surgical treatments directly administered. No external funding supported the present study.

\section{Search strategy}

An electronic search was performed on 31 September 2014 of the MEDLINE, EMBASE and 
Cochrane Central Register of Controlled Trials databases. The search terms used were "Anterior cruciate ligament" and "ACL" combined with the terms "revision", "reoperation" and "failure". Filters were utilised to access only those studies that investigated participants and were reported in English. No limitations were imposed on the date of publication. The reference section of each full-text obtained was scanned and handsearched for other possible inclusions to this study, which were not identified in the electronic database search.

\section{Eligibility criteria}

Studies were included if they met the following criteria: (1) randomised controlled trial (RCT), prospective or retrospective study, (2) the study reported the outcomes of both first or multiple revision ACL reconstruction, (3) the study reported at least one of: the return to any kind of sport rate, return to pre-injury sport level. When "return to pre-injury activity" was used as the outcome without mentioning the nature of the activity, the definition was considered too vague and therefore the study was excluded. The authors of the studies that met some but not all of the criteria were contacted for further information and to clarify whether the study was eligible or not.

\section{Study selection}

Two authors (AG and GMMM) independently reviewed the studies found during the database search. Articles were screened for eligibility based on a review of the title and abstract only. Any potential disagreement was resolved via consensus discussion between two independent reviewers, with a third reviewer (SZ) being consulted if the disagreement could not be resolved. When the eligibility was unclear from the title and abstract, the full text was obtained and assessed and again evaluated for eligibility.

\section{Data collection and analysis}

All the data regarding the names of authors, year of publication, number of patients treated, final follow-up duration and graft used for revision procedure were obtained and tabulated in an Excel extraction form to systematically extract the data. Regarding the sport-specific outcomes, details of all the patients who were still practising any kind of sport at final follow-up, and of all the patients who at final follow-up returned to the same level practised before the first ACL injury, were accurately extracted from each study and evaluated as primary outcomes. When possible, the subgroups of patients who returned to high level and competitive sport activity-according to clinical scores and definitions provided in each study - were also extracted.

The secondary outcomes relative to the final follow-up were the objective IKDC ${ }^{15} 16$ classification, knee laxity measured as side-to-side difference at the manual maximum displacement test measured in mm with KT-1000 or KT-2000 and results of the pivot-shift test. The percentage of participants with knee classified as category A (normal knee), B (nearly normal knee), $\mathrm{C}$ (abnormal knee) or D (severely abnormal knee) according to the IKDC form was calculated. Arithmetic averages of knee laxity and percentages of patients with a $<3,3-5$ or $>5 \mathrm{~mm}$ side to side difference at the manual maximum displacement test were calculated as well. The total number of patients with a pivot-shift test graded as normal (equal to the contralateral side), grade I (or glide, in case defined as a small and gentle sliding reduction), grade II (or clunk, in case of reduction movement easily appreciated) and grade III (gross, in case of an abnormal movement with a pronounced clunk) ${ }^{17} 18$ was calculated and expressed as a percentage for each groups. As for multiple revisions, the data regarding first revision were collected when available; otherwise, only data of the last revision were used for analysis.

\section{Quality assessment}

The studies included were assessed using a six-item checklist developed by Ardern et $a l^{1}$ for the evaluation of return to sport outcomes after primary ACL reconstruction. The six items assessed were: selection criteria described, representative population selected, participants' pre-injury sports participation level reported, data collected prospectively, demographic data reported and postoperative sports participation level compared with preinjury level. Items were scored as follows: one point for satisfaction of the criterion, no points for not satisfying the criterion. A score of zero was given if it was unclear whether the criterion had been satisfied or not. All articles were assessed independently by two assessors, disagreements being resolved by consensus, and third reviewer intervention in case of disagreement not being resolved. $\kappa$ Coefficient for inter-rater agreement was calculated as well. The purpose of the quality assessment in this meta-analysis was to identify characteristics of study quality and design common to all included studies, and to provide a qualitative description of these characteristics in the meta-analysis.

\section{Statistical analysis}

Statistical evaluation was performed using MedCalc (MedCalc software, Acacialaan 22, Ostend, Belgium). Data regarding return to sport, return to pre-injury sport level and return to high-level/competitive sports were combined via a proportion meta-analysis using a random effects model. $\mathrm{I}^{2}$ index was used to measure the amount of inconsistency in the results of the included studies. ${ }^{19}{ }^{20}$ A larger $\mathrm{I}^{2}$ index indicates that a greater amount of the variability in the results is due to the heterogeneity rather than to chance. ${ }^{19} 20$ The mean and 95\%CI was calculated for return to sport, return to pre-injury sport level and return to high level/competitive sport rates. Studies included in the meta-analyses were weighted according to sample size. Return to sport rate data from the individual studies were presented through forest-plot graphics.

The objective IKDC, KT-1000 or KT-2000 values and pivotshift results were presented as the number of patients and percentage of patients for each category. The anteroposterior knee laxity measured with KT-1000 or KT-2000 was presented also as mean value expressed in $\mathrm{mm}$.

\section{RESULTS}

The literature search yielded a total of 803 results. A flow diagram of the selection process is shown in figure 1. After duplicates were removed, 472 studies remained. Of these, 414 were excluded after reviewing title and abstract review, as involving mostly surgical technique descriptions, in-vitro, biomechanics or radiologic studies, or because were studies not related to revision ACL reconstruction. The full text of the remaining 58 studies were obtained and reviewed for eligibility. Thirty-five studies were excluded because they did not report the outcomes requested in the inclusion criteria (24 studies) or because they were narrative or systematic reviews (11 papers). Therefore, 23 studies were included in the final analysis. ${ }^{10-13}$ ${ }^{21-39}$ A summary of the population examined with clinical outcomes is presented in table 1 .

\section{Demographic data}

The 23 reviewed studies presented the results of 1090 patients at a mean follow-up of 5.3 years (range 1.0-13.2 years). When 
Figure 1 PRISMA flow diagram of the selection process.

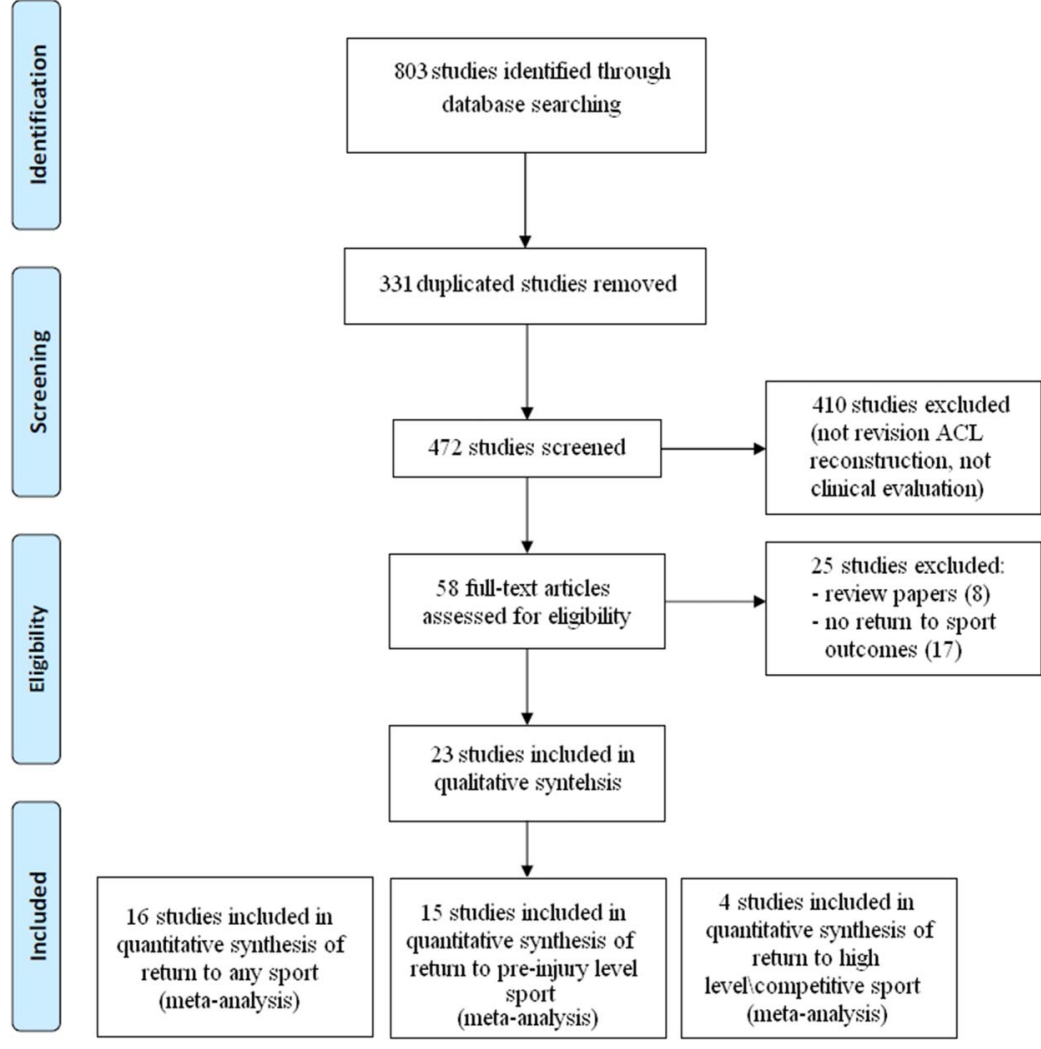

gender was reported, ${ }^{10} 111324262829333738$ revision ACL reconstruction was performed in 318 males (63\%) and 187 females (27\%). The mean age at revision surgery was 27.7 years (range 13-60 years), while the mean time from primary reconstruction and revision surgery, reported in 13 studies, ${ }^{10-12} 22-2427283031333437$ was 43.8 months (range 1-276 months). Most of the studies reported the results of a first revision procedure, while only four studies ${ }^{12} \quad 29 \quad 34 \quad 38$ reported at least one second-revision/third-revision procedure. Concerning the primary ACL lesions, 8\% sustained ACL repair and 3\% underwent an extra-articular procedure; when ACL reconstruction was instead performed, the primary graft to be used was bone patellar-tendon bone (BPTB) autograft in $57 \%$, hamstrings autograft in $16 \%$, other autografts in $2 \%$, synthetic grafts in 7\%, BPTB allograft in $2 \%$ and other allografts in $7 \%$.

Regarding the revision procedure, eight studies reported the results of one single specific graft, another eight used a combination of different autografts (6 studies) or allografts (2 studies), while the remaining seven reported the use of a heterogenous combination of allografts and autografts. Specifically, a BPTB autograft was used in 554 patients (50\%), a hamstrings autograft in 326 (29\%), a quadriceps tendon autograft in $71(6 \%)$, a BPTB allograft in 98 (9\%), an Achilles tendon allograft in 28 $(3 \%)$ and other allografts in $38(3 \%)$.

\section{Quality assessment}

Sixteen studies (69\%) were prospective case series, $4(17 \%)$ were retrospective case series while the remaining $3(14 \%)$ were comparative studies between primary and revision reconstruction or between different populations.

Regarding quality assessment, only 5 studies (22\%) fulfilled all the 6 items, while 9 (39\%) fulfilled 5 items. Three studies (13\%) fulfilled 4 items and 6 (26\%) fulfilled 3 items (table 2).
The item "selection criteria described" was fulfilled by all 23 studies (100\%), such as the "demographic data reported item". The "representative population selected" item was fulfilled by six studies (26\%) and the "data collected prospectively" item was fulfilled by 19 studies (83\%). Finally, the item "participants' pre-injury sports participation level reported" was fulfilled by 19 studies (83\%), while the "postoperative sports participation level compared with pre-injury level" was fulfilled by 15 studies (65\%).

The inter-rater agreement between the two reviewers regarding quality assessment was $\mathrm{k}=0.92$ (95\% CI 0.88 to 0.94$)$.

The definitions of the parameter used to assess the return to sport and pre-injury sport level were not consistent between all the studies and are described in table 2.

Only six studies reported the outcomes of athletic populations, with inconsistent description of the practised sports; the other studies involved mixed populations of both athletic and non-athletic patients, or did not provide information regarding the populations studied.

\section{Return to sport outcomes}

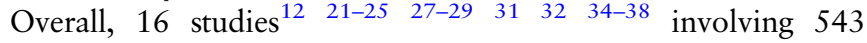
patients reported a rate of return to sport activity at any level at the final follow-up; the pooled rate was $84 \%$ (CI 78 to 89 ) (figure 2), $\mathrm{I}^{2}=65 \%(\mathrm{p}=0.0001)$.

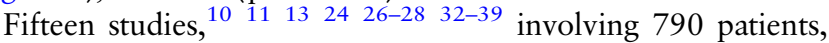
reported a rate of return to the same pre-injury sport level; the pooled rate was $52 \%$ (CI 36 to 67 ) (figure 3 ), $\mathrm{I}^{2}=95 \%$ $(\mathrm{p}=0.0001)$.

Four studies, ${ }^{26} 283032$ involving 186 patients, reported a rate of return to high-level/competitive sport; the pooled rate was $51 \%$ (CI 44 to 58 ) (figure 4$), \mathrm{I}^{2}=0 \%(\mathrm{p}=0.3921)$.

Only one study, ${ }^{12}$ involving 11 multiple revisions performed with reharvested ipsilateral or contralalateral BPTB, reported 
Table 1 Details of patient populations, follow-up duration, time from primary to revision ACL reconstruction, objective outcomes at final follow-up (objective IKDC, difference of laxity between indexed and healthy knee and pivot-shift test) and return to sport outcomes for each study

\begin{tabular}{|c|c|c|c|c|c|c|c|c|}
\hline Authors and year & Patients & $\begin{array}{l}\text { Follow-up } \\
\text { duration }\end{array}$ & $\begin{array}{l}\text { Age at revision } \\
\text { ACL reconstruction } \\
\text { (ACLR) }\end{array}$ & $\begin{array}{l}\text { Time from primary } \\
\text { to revision } A C L \\
\text { reconstruction }(A C L R)\end{array}$ & Final IKDC & Final laxity & $\begin{array}{l}\text { Final pivot-shift } \\
\mathrm{n}(\%)\end{array}$ & $\begin{array}{l}\text { Return to sport } \\
\text { n (\%) }\end{array}$ \\
\hline \multirow{8}{*}{$\begin{array}{l}\text { Noyes and Barber-Westin } \\
(1996)^{21}\end{array}$} & 65 & 3.5 years & 25 years & NA & NA & $3.4 \pm 4.8 \mathrm{~mm}$ & $34(52 \%)$ negative & $51(79 \%)$ Return to sport \\
\hline & & & & & & $30(53 \%)<3 \mathrm{~mm}$ & $24(37 \%)$ grade I & \\
\hline & & & & & & $17(30 \%) 3-5.5 \mathrm{~mm}$ & $4(6 \%)$ grade II & \\
\hline & & & & & & $10(17 \%)>5.5 \mathrm{~mm}$ & $3(5 \%)$ grade III & \\
\hline & 22 & 2.3 years & 27 years & NA & NA & $1.9 \pm 4.4 \mathrm{~mm}$ & $12(60 \%)$ negative & $16(80 \%)$ Return to sport \\
\hline & & & & & & $12(66 \%)<3 \mathrm{~mm}$ & $7(35 \%)$ grade I & \\
\hline & & & & & & $3(17 \%) 3-5.5 \mathrm{~mm}$ & $1(5 \%)$ grade II & \\
\hline & & & & & & $3(17 \%)>5.5 \mathrm{~mm}$ & $0(0 \%)$ grade III & \\
\hline \multirow[t]{4}{*}{ Johnson et al (1996) $)^{22}$} & 25 & 2.3 years & 25 years & 30 months & NA & $3.7 \pm 3.5 \mathrm{~mm}$ & $\begin{array}{l}20(80 \%) \text { negative- } \\
\text { grade I }\end{array}$ & $22(88 \%)$ Return to sport \\
\hline & $22 \mathrm{M} / 3 \mathrm{~F}$ & & & & & $5(20 \%)<2.5 \mathrm{~mm}$ & $5(20 \%)$ grade II & \\
\hline & & & & & & $11(44 \%) 3-5 \mathrm{~mm}$ & $0(0 \%)$ grade III & \\
\hline & & & & & & $9(36 \%) 5.5-10 \mathrm{~mm}$ & & \\
\hline \multirow{2}{*}{$\begin{array}{l}\text { Noyes and Barber-Westin } \\
(2001)^{23}\end{array}$} & 52 knees & 2.8 years & 27 years & NA & NA & $2.2 \pm 4.9 \mathrm{~mm}$ & NA & $40(72 \%)$ Return to sport \\
\hline & 51 patients & & & & & & & \\
\hline \multirow{3}{*}{$\begin{array}{l}\text { O'Shea and Shelbourne } \\
(2002)^{12}\end{array}$} & 11 & 4.1 years & 24 years & 71 months & NA & $1.6 \pm 1.2 \mathrm{~mm}$ & NA & $11(100 \%)$ Return to sport \\
\hline & & & & & & $11(100 \%)<3 \mathrm{~mm}$ & & $5.1 \pm 2.2$ weeks to light sports \\
\hline & & & & & & & & $5.4 \pm 1.5$ months to full sport \\
\hline \multirow[t]{4}{*}{ Taggart et al $(2004)^{24}$} & 20 & 3.4 years & 30 years & 49 months & NA & $3(15 \%)<1 \mathrm{~mm}$ & $3(15 \%)$ negative & $8(40 \%)$ Same pre-injury sport level \\
\hline & $14 \mathrm{M} / 6 \mathrm{~F}$ & & & & & $5(25 \%) 1-3 \mathrm{~mm}$ & $9(45 \%)$ grade I & $6(30 \%)$ Lower level \\
\hline & & & & & & $5(25 \%) 3-5 \mathrm{~mm}$ & $4(20 \%)$ grade II & \\
\hline & & & & & & $7(35 \%)>5 \mathrm{~mm}$ & $4(20 \%)$ grade III & \\
\hline \multirow[t]{5}{*}{ Carson et al $(2004)^{25}$} & 43 & Minimum 2 years & NA & NA & NA & $2.9 \mathrm{~mm}$ & $37(86 \%)$ negative & $12(28 \%)$ Sport without limitation \\
\hline & & & & & & $20(47 \%)<3 \mathrm{~mm}$ & & $20(46 \%)$ Sport with limitation \\
\hline & & & & & & $13(30 \%) 3-4 \mathrm{~mm}$ & & \\
\hline & & & & & & $6(14 \%) 4-5 \mathrm{~mm}$ & & \\
\hline & & & & & & $4(9 \%)>5 \mathrm{~mm}$ & & \\
\hline \multirow[t]{5}{*}{$0^{\prime}$ Neill $(2004)^{26}$} & 48 & 7.5 years & 33 years & 60 months & $20(42 \%) \mathrm{A}$ & $10(21 \%) 0 \mathrm{~mm}$ & NA & $36(75 \%)$ Same pre-injury sport level \\
\hline & $28 \mathrm{M} / 20 \mathrm{~F}$ & & & & $20(42 \%) \mathrm{B}$ & $22(46 \%) 0-2 \mathrm{~mm}$ & & 21 (44\%) High impact activities \\
\hline & & & & & $6(12 \%) C$ & $3(6 \%) 2-3 \mathrm{~mm}$ & & \\
\hline & & & & & $2(4 \%) D$ & $10(21 \%) 3-5 \mathrm{~mm}$ & & \\
\hline & & & & & & $3(6 \%)>5 \mathrm{~mm}$ & & \\
\hline \multirow[t]{4}{*}{ Grossman et al $(2005)^{10}$} & 29 & 5.6 years & 30.2 years & 56 months & $17(58 \%) \mathrm{A}$ & $2.8 \mathrm{~mm}$ & $27(93 \%)$ negative & $20(68 \%)$ Same pre-injury sport level \\
\hline & $22 \mathrm{M} / 7 \mathrm{~F}$ & & & & $8(28 \%)$ B & & $2(7 \%)$ grade I & \\
\hline & & & & & $4(14 \%) C$ & & $0(0 \%)$ grade II & \\
\hline & & & & & $0(0 \%) \mathrm{D}$ & & $0(0 \%)$ grade III & \\
\hline
\end{tabular}




\begin{tabular}{|c|c|c|c|c|c|c|c|c|}
\hline Authors and year & Patients & $\begin{array}{l}\text { Follow-up } \\
\text { duration }\end{array}$ & $\begin{array}{l}\text { Age at revision } \\
\text { ACL reconstruction } \\
\text { (ACLR) }\end{array}$ & $\begin{array}{l}\text { Time from primary } \\
\text { to revision } A C L \\
\text { reconstruction (ACLR) }\end{array}$ & Final IKDC & Final laxity & $\begin{array}{l}\text { Final pivot-shift } \\
\mathbf{n}(\%)\end{array}$ & $\begin{array}{l}\text { Return to sport } \\
\text { n (\%) }\end{array}$ \\
\hline \multirow[t]{4}{*}{ Thomas et al $(2005)^{13}$} & 49 & 6.2 years & 35.4 years & NA & $12(24 \%) \mathrm{A}$ & $1.9 \pm 2.4 \mathrm{~mm}$ & $43(88 \%)$ negative & $0(0 \%)$ Same pre-injury sport level \\
\hline & $37 \mathrm{M} / 12 \mathrm{~F}$ & & & & $28(58 \%)$ B & $46(94 \%) 0-2 \mathrm{~mm}$ & $5(10 \%)$ grade I & \\
\hline & & & & & $8(16 \%) C$ & $1(2 \%) 3-4 \mathrm{~mm}$ & $1(2 \%)$ grade II & \\
\hline & & & & & $1(2 \%) D$ & $2(4 \%)>5 \mathrm{~mm}$ & $0(0 \%)$ grade III & \\
\hline \multirow[t]{4}{*}{ Ferretti et al $(2006)^{27}$} & 28 & 5.0 years & 34 years & 60 months & $15(53 \%) \mathrm{A}$ & $2.5 \pm 1.8 \mathrm{~mm}$ & $15(54 \%)$ negative & $6(20 \%)$ Same pre-injury sport level \\
\hline & & & & & $11(39 \%)$ B & $20(71 \%)$ 0-3 mm & $11(39 \%)$ grade I & 9 (30\%) Lower level \\
\hline & & & & & $2(8 \%) C$ & $6(21 \%) 3-5 \mathrm{~mm}$ & $2(7 \%)$ grade II & $30(100 \%)$ Return to sport \\
\hline & & & & & $0(0 \%) D$ & $2(8 \%)>5 \mathrm{~mm}$ & $0(0 \%)$ grade III & \\
\hline \multirow[t]{4}{*}{ Garofalo et al $(2006)^{28}$} & 28 & 4.2 years & 27 years & 26 months & $5(18 \%) A$ & $3.1 \pm 2.0 \mathrm{~mm}$ & $21(75 \%)$ negative & 26 (93\%) Same pre-injury sport level \\
\hline & $16 \mathrm{M} / 12 \mathrm{~F}$ & & & & $21(75 \%) \mathrm{B}$ & $18(65 \%)<3 \mathrm{~mm}$ & $7(25 \%)$ grade I & $18(64 \%)$ High competitive \\
\hline & & & & & $2(7 \%) C$ & $9(32 \%) 3-5 \mathrm{~mm}$ & $0(0 \%)$ grade II & $6(21 \%)$ Recreational \\
\hline & & & & & $0(0 \%) D$ & $\begin{array}{l}1(3 \%) 6-10 \mathrm{~mm} \\
0(0 \%)>10 \mathrm{~mm}\end{array}$ & $0(0 \%)$ grade III & 2 (7\%) Light sport \\
\hline \multirow{4}{*}{$\begin{array}{l}\text { Noyes and Barber-Westin } \\
(2006)^{29}\end{array}$} & 21 & 4.1 years & 33 years & NA & $5(24 \%) A$ & $2.1 \pm 2.2 \mathrm{~mm}$ & $10(48 \%)$ negative & $15(71 \%)$ Return to sport \\
\hline & $15 \mathrm{M} / 6 \mathrm{~F}$ & & & & $11(52 \%) \mathrm{B}$ & $8(38 \%)<3 \mathrm{~mm}$ & $7(33 \%)$ grade I & \\
\hline & & & & & $3(14 \%) C$ & $7(33 \%) 3-5 \mathrm{~mm}$ & $3(14 \%)$ grade II & \\
\hline & & & & & $2(10 \%) D$ & $4(19 \%)>5 \mathrm{~mm}$ & $1(5 \%)$ grade III & \\
\hline \multirow[t]{4}{*}{ Salmon et al $(2006)^{30}$} & 50 & 7.4 years & 27 years & 36 months & $33(73 \%) \mathrm{A} / \mathrm{B}$ & $2.5 \mathrm{~mm}$ & $31(69 \%)$ negative & $26(58 \%)$ Return to level I-II activity \\
\hline & & & & & $12(27 \%) C / D$ & $17(50 \%)<3 \mathrm{~mm}$ & $14(31 \%)$ grade I & \\
\hline & & & & & & $17(50 \%) 3-5 \mathrm{~mm}$ & $0(0 \%)$ grade II & \\
\hline & & & & & & $0(0 \%)>5 \mathrm{~mm}$ & $0(0 \%)$ grade III & \\
\hline \multirow[t]{4}{*}{ Battaglia et al $(2007)^{31}$} & 63 & 6.1 years & 31 years & 61 months & NA & $3.9 \pm 2.4 \mathrm{~mm}$ & NA & $42(66 \%)$ Return to sport \\
\hline & $36 \mathrm{M} / 27 \mathrm{~F}$ & & & & & $30(48 \%)<3 \mathrm{~mm}$ & & \\
\hline & & & & & & $20(31 \%) 3-5 \mathrm{~mm}$ & & \\
\hline & & & & & & $10(21 \%)>5 \mathrm{~mm}$ & & \\
\hline \multirow[t]{4}{*}{ Denti et al $(2008)^{32}$} & 60 & 3.5 years & 31 years & NA & $18(36 \%) \mathrm{A}$ & $28(56 \%)<3 \mathrm{~mm}$ & NA & $47(78 \%)$ Same pre-injury sport level \\
\hline & & & & & $23(46 \%)$ B & $17(34 \%) 3-5 \mathrm{~mm}$ & & 8 (14\%) Lower level \\
\hline & & & & & $9(18 \%) C$ & $5(10 \%) 6-10 \mathrm{~mm}$ & & $30(50 \%)$ High competitive activities \\
\hline & & & & & $0(0 \%) D$ & $0(0 \%)>10 \mathrm{~mm}$ & & \\
\hline \multirow[t]{4}{*}{ Diamantopoulos et al (2008) ${ }^{33}$} & 107 & 6.0 years & 39 years & 60 months & 79 (74\%) A & $0.9 \pm 1.1 \mathrm{~mm}(\mathrm{KT})$ & $79(74 \%)$ negative & $39(36 \%)$ Same pre-injury sport level \\
\hline & $64 \mathrm{M} / 43 \mathrm{~F}$ & & & & $17(16 \%) \mathrm{B}$ & & $17(16 \%)$ grade I & \\
\hline & & & & & $11(10 \%) C$ & & $11(10 \%)$ grade II & \\
\hline & & & & & $0(0 \%) D$ & & $0(0 \%)$ grade III & \\
\hline \multirow[t]{7}{*}{ Wegrzyn et al (2009) } & 10 & 3.2 years & 30 years & 26 months & $4(40 \%) \mathrm{A}$ & $1.3 \pm 1.9 \mathrm{~mm}$ & NA & 1st revision: \\
\hline & $8 \mathrm{M} / 2 \mathrm{~F}$ & & & & $5(50 \%) B$ & $9(90 \%)<5 \mathrm{~mm}$ & & $5(50 \%)$ Same pre-injury sport level \\
\hline & & & & & $1(10 \%) C$ & $1(10 \%)>5 \mathrm{~mm}$ & & 4 (40\%) Lower level \\
\hline & & & & & $0(0 \%) D$ & & & \\
\hline & & & & & & & & 2nd revision: \\
\hline & & & & & & & & $2(20 \%)$ Same pre-injury sport level \\
\hline & & & & & & & & $4(40 \%)$ Lower level \\
\hline
\end{tabular}


Table 1 Continued

\begin{tabular}{|c|c|c|c|c|c|c|c|c|}
\hline Authors and year & Patients & $\begin{array}{l}\text { Follow-up } \\
\text { duration }\end{array}$ & $\begin{array}{l}\text { Age at revision } \\
\text { ACL reconstruction } \\
\text { (ACLR) }\end{array}$ & $\begin{array}{l}\text { Time from primary } \\
\text { to revision ACL } \\
\text { reconstruction (ACLR) }\end{array}$ & Final IKDC & Final laxity & $\begin{array}{l}\text { Final pivot-shift } \\
\mathrm{n}(\%)\end{array}$ & $\begin{array}{l}\text { Return to sport } \\
\mathrm{n}(\%)\end{array}$ \\
\hline \multirow[t]{4}{*}{ Muneta et al (2010) $)^{35}$} & 21 & 3.3 years & 27 years & NA & NA & $1.7 \pm 1.8 \mathrm{~mm}$ & 14 (78\%) negative & $13(76 \%)$ Same pre-injury sport level \\
\hline & $14 \mathrm{M} / 7 \mathrm{~F}$ & & & & & & $3(17 \%)$ grade I & $18(86 \%)$ Return to sport \\
\hline & & & & & & & $1(5 \%)$ grade II & \\
\hline & & & & & & & $0(0 \%)$ grade III & \\
\hline \multirow[t]{4}{*}{ Reinhardt et al (2012) ${ }^{36}$} & 21 & 3.0 years & 16.9 years & NA & NA & NA & $20(95 \%)$ negative & $11(52 \%)$ Same pre-injury sport level \\
\hline & & & & & & & 1 (5\%) grade I & $20(95 \%)$ Return to sport \\
\hline & & & & & & & $0(0 \%)$ grade II & \\
\hline & & & & & & & $0(0 \%)$ grade III & \\
\hline \multirow[t]{4}{*}{ Franceschi et al $(2013)^{37}$} & 30 & 6.8 years & 29.1 years & 34 months & $27(90 \%) A / B$ & $3.1 \mathrm{~mm}$ & $25(83 \%)$ Negative & $20(67 \%)$ Same pre-injury sport level \\
\hline & $19 \mathrm{M} / 11 \mathrm{~F}$ & & & & $3(10 \%) C$ & & $5(17 \%)$ grade I & 7 (25\%) Lower activity \\
\hline & & & & & $0(0 \%) \mathrm{D}$ & & $0(0 \%)$ grade II & \\
\hline & & & & & & & $0(0 \%)$ grade III & \\
\hline \multirow[t]{4}{*}{ Gifstad et al (2013) $)^{11}$} & 56 & 7.5 years & 26.5 years & 38 months & NA & $3.3 \pm 2.7 \mathrm{~mm}$ & $\begin{array}{l}45(80 \%) \text { negative- } \\
\text { grade I }\end{array}$ & 7 (13\%) Same pre-injury sport level \\
\hline & $25 \mathrm{M} / 31 \mathrm{~F}$ & & & & & $28(52 \%)<3 \mathrm{~mm}$ & $11(20 \%)$ grade II-III & \\
\hline & & & & & & $17(23 \%) 3-5 \mathrm{~mm}$ & & \\
\hline & & & & & & $8(15 \%)>5 \mathrm{~mm}$ & & \\
\hline \multirow[t]{4}{*}{ Buda et al $(2013)^{38}$} & 24 & 3.3 years & NA & NA & $4(17 \%) A$ & $3.1 \pm 1.1 \mathrm{~mm}$ & $4(17 \%)$ negative & $17(71 \%)$ Same pre-injury sport level \\
\hline & $24 \mathrm{M} / \mathrm{OF}$ & & & & $16(67 \%) B$ & & $18(75 \%)$ grade I & 3 (13\%) Lower level \\
\hline & & & & & $2(8 \%) C$ & & $2(8 \%)$ grade II & \\
\hline & & & & & $2(8 \%) D$ & & $0(0 \%)$ grade III & \\
\hline Shelbourne et al (2014) ${ }^{39}$ & 259 & 7.2 years & 22.4 years & NA & NA & $2.3 \pm 1.8 \mathrm{~mm}$ & NA & $\begin{array}{l}178(69 \%) \text { Same pre-injury sport } \\
\text { level }\end{array}$ \\
\hline
\end{tabular}

Pre-injury level is referred to the initial injury before primary $\mathrm{ACL}$ reconstruction. Values are expressed as the mean or absolute number and percentage, according to variable.

F, females; M, males; IKDC, International Knee Documentation Committee; NA, not applicable. 
Table 2 Details of study design, quality assessment, mixed or athletic population included in each study and the definition of return to sport extracted from the text of each study, with the scale to measure activity level (when used)

\begin{tabular}{|c|c|c|c|c|}
\hline Authors and year & Study design & $\begin{array}{l}\text { Quality } \\
\text { assessment }\end{array}$ & Population & Return to sport evaluation \\
\hline Noyes and Barber-Westin $(1996)^{21}$ & Prospective observational study & 5 & Mixed & Sport activity preoperatively and at follow-up \\
\hline Johnson et al (1996) ${ }^{22}$ & Prospective observational study & 5 & Mixed & Sport activity frequency preoperatively and postoperatively \\
\hline Noyes and Barber-Westin $(2001)^{23}$ & Prospective observational study & 3 & Mixed & $\begin{array}{l}\text { Return to sports } \\
\text { Cincinnati knee rating system }\end{array}$ \\
\hline O'Shea and Shelbourne $(2002)^{12}$ & Prospective observational study & 3 & Mixed & Return to sports \\
\hline Taggart et al (2004) $)^{24}$ & Retrospective case series & 5 & Athletes & $\begin{array}{l}\text { Return to same pre-injury sport } \\
\text { Return to sport at any level }\end{array}$ \\
\hline Carson et al $(2004)^{25}$ & Retrospective case series & 3 & Mixed & Return to sports \\
\hline O'Neill $(2004)^{26}$ & Prospective observational study & 5 & Mixed & $\begin{array}{l}\text { Return to same pre-injury sport } \\
\text { Return to pivotinglcuttinglcontact }\end{array}$ \\
\hline Grossman et al $(2005)^{10}$ & Retrospective case series & 3 & Mixed & $\begin{array}{l}\text { Return to same pre-injury activity and sport } \\
\text { Return to collegiate sport }\end{array}$ \\
\hline Thomas et al $(2005)^{13}$ & Prospective comparative study & 4 & Mixed & Return to original level of sport activity \\
\hline Ferretti et al $(2006)^{27}$ & Prospective observational study & 6 & Athletes & $\begin{array}{l}\text { Return to same pre-injury sports } \\
\text { Return to sports }\end{array}$ \\
\hline Garofalo et al (2006) ${ }^{28}$ & Prospective observational study & 6 & Athletes & $\begin{array}{l}\text { Return to previous sport activity } \\
\text { IKCD activity score }\end{array}$ \\
\hline Noyes and Barber-Westin $(2006)^{29}$ & Prospective observational study & 3 & Mixed & $\begin{array}{l}\text { Return to sport } \\
\text { Cincinnati knee rating system }\end{array}$ \\
\hline Salmon et al $(2006)^{30}$ & Prospective observational study & 4 & Mixed & IKDC activity score \\
\hline Battaglia et al $(2007)^{31}$ & Prospective observational study & 3 & Mixed & $\begin{array}{l}\text { Return to sport } \\
\text { IKDC activity score }\end{array}$ \\
\hline Denti et al $(2008)^{32}$ & Prospective observational study & 5 & Mixed & Return to same pre-injury sports \\
\hline Diamantopoulos et al (2008) $)^{33}$ & Prospective observational study & 5 & Mixed & Return to same pre-injury sport activity \\
\hline Wegrzyn et al (2009) & Retrospective case series & 4 & Mixed & Return to same pre-injury sports \\
\hline Muneta et al $(2010)^{35}$ & Retrospective comparative study & 5 & Mixed & Return to sport activity equal to pre-injury level \\
\hline Reinhardt et al (2012) ${ }^{36}$ & Prospective observational study & 6 & Athletes & $\begin{array}{l}\text { Return to same or higher activitylsport level } \\
\text { IKDC activity score }\end{array}$ \\
\hline Franceschi et al $(2013)^{37}$ & Prospective observational study & 5 & Mixed & $\begin{array}{l}\text { Return to pre-injury sport level } \\
\text { Return to lower non-impact sports }\end{array}$ \\
\hline Gifstad et al (2013) $)^{11}$ & Prospective observational study & 5 & Mixed & Return to same or better pre-injury sport level \\
\hline Buda et al $(2013)^{38}$ & Prospective observational study & 6 & Athletes & $\begin{array}{l}\text { Return to same pre-injury sport level } \\
\text { Sport resumption at lower level }\end{array}$ \\
\hline Shelbourne et al (2014) $)^{39}$ & Prospective comparative study & 6 & Athletes & Return to same sport at previous level \\
\hline
\end{tabular}

IKDC, International Knee Documentation Committee.

the time to return to sport. Return to light sport was achieved after $5.1 \pm 2.2$ weeks after revision, while return to full sport was achieved after $5.4 \pm 1.5$ months.

Only three studies, ${ }^{21} 28{ }^{31}$ involving 174 patients, reported the main reason for not returning to sport after ACL revision surgery: 31 patients (69\%) were not able to return to sport because of knee-related problems, 10 (22\%) because of the fear of re-injury while 4 (9\%) were unable to do so for other reasons.

\section{Secondary outcomes}

Twelve studies ${ }^{10} \quad 13 \quad 26-30 \quad 32-343738$ reported the IKDC results in 392 patients. At final follow-up, 177 patients (45\%) had knee function rated as normal (A), $160(41 \%)$ as nearly normal (B), $48(12 \%)$ as abnormal (C) and $7(2 \%)$ as severely abnormal (D).

Twenty-two studies ${ }^{10-13}$ 21-35 37-39 reported laxity evaluation at the final follow-up of 1069 patients, reporting a mean final value of $2.6 \mathrm{~mm}$ (range $-4-6 \mathrm{~mm}$ ). When reported, 325 patients $(58 \%)$ presented a value of side-to-side difference at manual maximum displacement test $<3 \mathrm{~mm}, 169 \quad(30 \%)$ between 3 and $5 \mathrm{~mm}$, and $69(12 \%)>5 \mathrm{~mm}$.

Sixteen studies ${ }^{10} \quad 11 \quad 13 \quad 21 \quad 22 \quad 24 \quad 26-30 \quad 33 \quad 35-38$ reported the results of the postoperative Pivot-Shift test in 515 patients. The test was considered negative in 338 patients (67\%), while it was graded as grade I in 140 (26\%), grade II in $29(5 \%)$ and grade III in $8(2 \%)$.

Therefore, $337 / 392(86 \%)$ patients presented a normal or nearly normal knee (group A and B according to objective IKDC), 494/563 (88\%) had normal values of static anteroposterior laxity (side to side difference $<5 \mathrm{~mm}$ at KT-1000/2000) and $478 / 515(93 \%)$ had a normal or nearly normal rotatory laxity (normal or grade I pivot-shift test).

\section{DISCUSSION}

The main findings of this meta-analysis are that $84 \%$ of patients returned to sport after revision ACL reconstruction, while 52\% returned to the same pre-injury sport level and 51\% returned to high-level/competitive sports.

The return to sport after both primary and revision ACL reconstruction represents a complex outcome to evaluate. First of all, a great inconsistency in reporting the results was found in most of the studies, as a clear and unequivocal definition of return to sport was not provided. For example, the concept of "return to pre-injury sport level" presented a not unequivocal interpretation in most of the studies, especially regarding the kind of sport and its level (amateur, competitive, elite), possibly 
Figure 2 Forest-plot graphic of return to sport outcome; \%, mean percentage; $\mathrm{Cl}, 95 \% \mathrm{Cls}$.

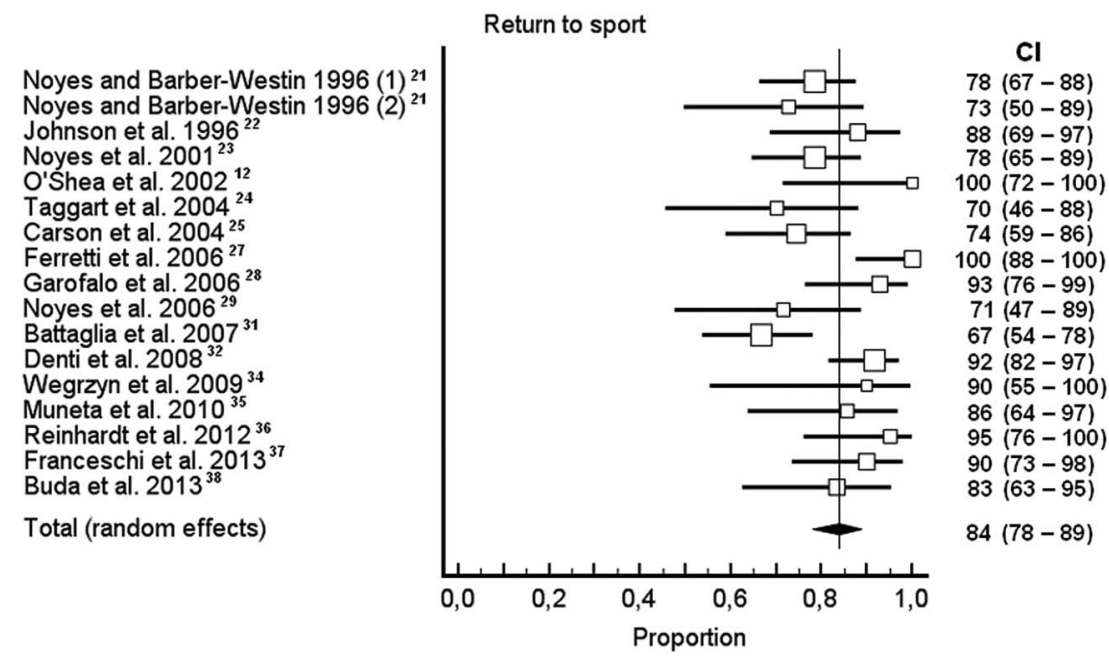

contributing to the production of high variability of the results reported in this meta-analysis. In fact, the latter outcome was mostly assessed with the sentence "patients returned to the same pre-injury sport activity" or "were able to perform sport equal to pre-injury level", without mentioning the type of sport, level and intensity. Moreover, only a few studies reported the specific sport performed by each of the patients, thus possibly creating a bias because it could be speculated that patients who perform less demanding activities like tennis or running could hypothetically return to sport easier than patients performing pivoting sports like soccer, basketball and handball.

With the available data obtained from this meta-analysis, the return to any sport outcome was similar to what was reported after primary ACL reconstruction; ${ }^{1}$ while the return to preinjury sport level resulted lower compared to primary ACL reconstruction. This could also be due to the higher age at revision ACL reconstruction. In fact, the revision ACL reconstruction could be performed in patients near to 40 years of age. Therefore it could be difficult for them to return to perform the same activity of long time before, when they had the primary ACL injury, and this is not always necessarily due to reasons related to knee performance. Moreover it should be noted that the activity before a primary ACL lesion was used as a landmark in most of the studies. Conversely, the unreported activity level prior to primary ACL reconstruction failure would have probably pointed out the real impact of revision procedure on sport activity, or at least provided adjunctive information regarding the changes of sport practice along the whole follow-up from the very first injury to final evaluation.

Regarding the impact of graft type in the return to sport outcomes, the analysis of the data from the current literature produced inconsistent results. This is mostly due to the lack of well-designed RCTs and to the high heterogeneity in graft choice, between and within each single study. This situation reflects the complexity of revision surgery, such as graft availability derived from previous reconstructions, the status of the contralateral knee, the availability of allograft tissue or the need for a graft with bone stock, which often make the graft choice a necessity rather than a preference. Most of authors used the unharvested BPTB or hamstrings as first choice, while reserving quadriceps tendon autograft, contralateral grafts or various allografts only in case of repeated revision, combined posterior cruciate ligament or posterolateral corner reconstruction, previous multiligament reconstruction or other particular extraordinary circumstances. Therefore only a few studies included revision ACL reconstruction with a single specific graft, making it impossible to compare sport-specific outcomes based on graft choice.

A wide discrepancy is noted between successful results of revision ACL reconstruction based on objective evaluation and sport-related outcomes; normal or nearly normal results were reported in $86 \%, 88 \%$ and $93 \%$ for objective IKDC, anteroposterior knee laxity and Pivot-Shift test, respectively, against a rate
Figure 3 Forest-plot graphic of return to pre-injury sport level; \%, mean percentage; $\mathrm{Cl}, 95 \% \mathrm{Cls}$.
Return to same pre-injurty sport level
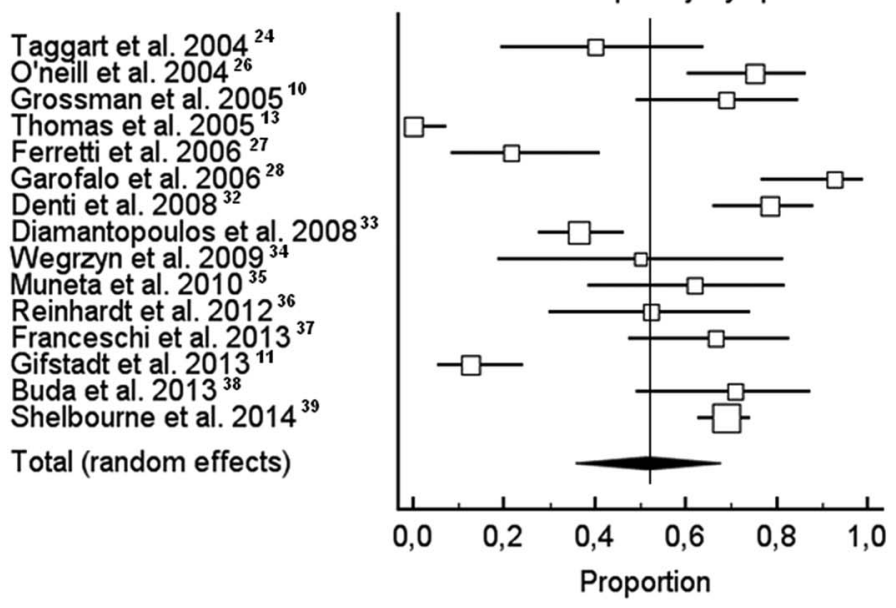

\section{CI}

$40(19-64)$

$75(60-86)$

$69(49-85)$

$0(0-7)$

$21(8-41)$

$93(76-99)$

$78(59-86)$

$36(27-46)$

$50(19-81)$

$62(38-82)$

$52(30-74)$

$66(47-83)$

$13(5-24)$

$71(48-87)$

$69(63-74)$

$52(36-67)$ 


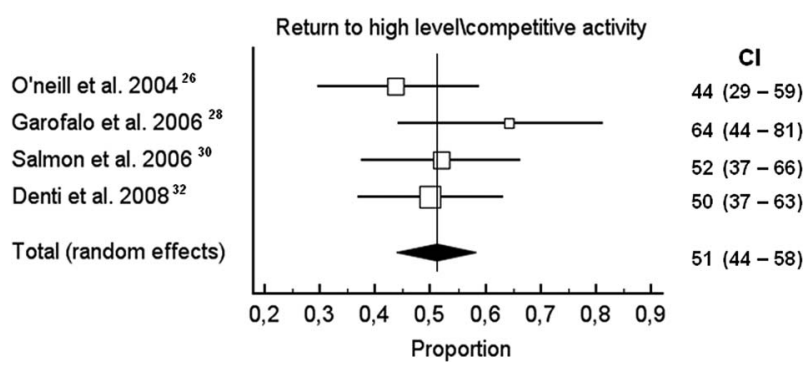

Figure 4 Forest-plot graphic of return to high-level/competitive sport; $\%$, mean percentage; $\mathrm{Cl}, 95 \% \mathrm{Cls}$.

of $52 \%$ of return to pre-injury sport level. The measurement of sport participation represents a relevant issue, especially in athletes and high-level patients, probably superior to objective tools such as knee stability, ROM and muscle strength, because sport participation is more meaningful for them in their daily lives. The reasons for the discrepancy between objective and sport-related outcomes should be evaluated and addressed, as probably in not all cases the inability to return to the same preinjury sport level was due to the indexed knee problems. In contrast, fear of re-injury, priorities and personality could influence the decision to return to sport. ${ }^{3}{ }^{40}$ It has in fact been previously demonstrated that issues unrelated to surgical outcomes, such as lack of motivations, reduced confidence, fear of sport incompetence or lower priority on sport due to family/work-related reasons, have negative effects on the return to pre-injury sport level. ${ }^{3}$ Such evidence, besides representing another potential contributing factor to the high variability of results, could point out the necessity of psychological interventions integrated in the postoperative rehabilitation process to possibly try to improve the outcomes of revision ACL reconstruction, as already suggested for primary ACL reconstruction. ${ }^{41}$ Moreover, poor surgery, inadequate rehabilitation and impairment of muscle performance, isokinetic strength and functional tests could also affect the ability to return to sport. ${ }^{4}$

This meta-analysis presents some strong limitations. First of all, it ought to be mentioned that the return to sport rate considered in this study actually represents the number of patients who are still able to perform sports at the final follow-up. It is plausible that, during the considered follow-up, some patients were able to return to sport after revision ACL reconstruction and rehabilitation, but that several reasons caused them to eventually abandon the practice shortly afterwards. The actual return to sports rate would thus include all patients/athletes being able to resume sports at any time after ACL revision surgery, irrespective of whether they were still active or not at the time of follow-up. However, the 'return to sports rate' and the 'still active at follow-up rate' were not reported separately in the included studies, thus making an adequate analysis impossible as well as potentially generating misinterpretation of the results. Therefore, the reader should be aware of this major issue and take a conservative approach when drawing conclusions from this meta-analysis.

Another major limitation was the high heterogeneity of the studies included, particularly regarding patients' characteristics and surgical treatment, which did not allow an analysis of subgroups regarding sport participation and objective outcomes. This is true also for first or second or third revision ACL reconstruction, as only two studies exclusively evaluated this population, making further analysis impossible. Furthermore, the dishomogeneous and equivocal definition of return to sport and return to pre-injury sport level could have created a bias when interpreting the results. Also, owing to the lack of studies with a follow-up of less than 2 years, it was impossible to compare the short-term results of revision ACL reconstruction with the medium-term and long-term results, as already performed by Ardern et $a l^{1}$ when evaluating primary ACL reconstruction. This made it impossible to monitor the sport practice from short-term to long-term follow-up, and to detect potential decreases of sport activity with the passage of time. Finally, the evaluation of subjectively measured parameters, such as Pivot-shift test and IKDC score, could have produced a concrete bias regardless of whether independent examiners were involved in the patient's evaluation, because these objective measures could lack sensitivity to detect subtle differences in function after revision ACL reconstruction. Unfortunately, this issue could not have been investigated adequately due to the great inconsistency in reporting the details regarding the examiner(s) in each study. On the other hand, the evaluation of an objective parameter such as knee laxity determined with KT-1000/2000, despite the lack of inter-rater reliability across different studies, could provide a concrete measure of postoperative outcomes.

Future high-quality RCTs or prospective case series with homogeneous populations, especially regarding graft choice and surgical techniques, could improve the knowledge and understanding of revision ACL reconstruction outcomes. Moreover, the use of a clear definition of sport-related outcomes, such as return to pre-injury sport level, return to training or competitive sport, explicit presentation of the type of sport and level practised before ACL injury, at the end of rehabilitation, and at the final follow-up, could avoid a misleading interpretation of revision ACL reconstruction outcomes and better understanding of the effective ability to perform sport activity after this kind of surgical procedure.

\section{What is known about the topic?}

- Revision $\mathrm{ACL}$ reconstruction is an increasingly performed procedure due to the high number of primary $\mathrm{ACL}$ reconstructions and failures.

- Revision ACL reconstruction is able to restore knee stability and improve knee function.

- Return to sport after revision ACL reconstruction has not been deeply investigated.

- Wide ranges of return to sport are present in the current literature.

\section{What are the new findings?}

- $84 \%$ of patients who had revision $\mathrm{ACL}$ reconstruction returned to any kind of sport activity.

- $52 \%$ of patients returned to their pre-injury sport level.

- There was a higher percentage of patients with normal or nearly normal knees at final follow-up, compared to the proportion who returned to sport.

- A homogeneous definition of return to sport when dealing with revision $A C L$ reconstruction needs to be clarified in future studies. 


\section{CONCLUSIONS}

On the basis of the data of the present meta-analysis, $84 \%$ of patients returned to sport after revision ACL reconstruction, while $52 \%$ returned to the same pre-injury sport level. Normal, or nearly normal, objective results were reported in $86 \%, 88 \%$ and $93 \%$ for objective IKDC, anteroposterior knee laxity and Pivot-Shift test, respectively. The high heterogeneity of patient populations and sport-related outcome definitions represent the major biases when evaluating return to sport outcomes after ACL reconstruction outcomes.

Contributors AG, GMMM and SZ performed a database search and review of articles. MPN and SDV performed data collection and an analysis of the results. MM wrote the paper and critically evaluated the results.

Competing interests None declared.

Provenance and peer review Not commissioned; externally peer reviewed.

\section{REFERENCES}

1 Ardern $\mathrm{CL}$, Webster $\mathrm{KE}$, Taylor NF, et al. Return to sport following anterior cruciate ligament reconstruction surgery: a systematic review and meta-analysis of the state of play. Br J Sports Med 2011:45:596-606.

2 Ardern $\mathrm{CL}$, Taylor NF, Feller JA, et al. Psychological responses matter in returning to preinjury level of sport after anterior cruciate ligament reconstruction surgery. Am J Sports Med 2013;41:1549-58.

3 Tjong VK, Murnaghan ML, Nyhof-Young JM, et al. A qualitative investigation of the decision to return to sport after anterior cruciate ligament reconstruction: to play or not to play. Am J Sports Med 2014;42:336-42.

4 Thomeé R, Kaplan Y, Kvist J, et al. Muscle strength and hop performance criteria prior to return to sports after $A C L$ reconstruction. Knee Surg Sports Traumatol Arthrosc 2011;19:1798-805.

5 Bizzini M, Silvers HJ. Return to competitive football after major knee surgery: more questions than answers? J Sports Sci 2014;32:1209-16.

6 Kvist J. Rehabilitation following anterior cruciate ligament injury: current recommendations for sports participation. Sports Med 2004;34:269-80.

7 Warner SJ, Smith MV, Wright RW, et al. Sport-specific outcomes after anterior cruciate ligament reconstruction. Arthroscopy 2011;27:1129-34.

8 Wright RW, Gill CS, Chen L, et al. Outcome of revision anterior cruciate ligament reconstruction: a systematic review. J Bone Joint Surg Am 2012;94:531-6.

9 Gianotti SM, Marshall SW, Hume PA, et al. Incidence of anterior cruciate ligament injury and other knee ligament injuries: a national population-based study. J Sci Med Sport 2009;12:622-7.

10 Grossman $\mathrm{MG}$, ElAttrache NS, Shields $\mathrm{CL}$, et al. Revision anterior cruciate ligament reconstruction: three- to nine-year follow-up. Arthroscopy 2005;21:418-23.

11 Gifstad T, Drogset JO, Viset A, et al. Inferior results after revision ACL reconstructions: a comparison with primary $\mathrm{ACL}$ reconstructions. Knee Surg Sports Traumatol Arthrosc 2013;21:2011-18.

12 O'Shea JJ, Shelbourne KD. Anterior cruciate ligament reconstruction with a reharvested bone-patellar tendon-bone graft. Am J Sports Med 2002;30:208-13.

13 Thomas NP, Kankate R, Wandless F, et al. Revision anterior cruciate ligament reconstruction using a 2 -stage technique with bone grafting of the tibial tunnel. Am J Sports Med 2005;33:1701-9.

14 Moher D, Liberati A, Tetzlaff J, et al., PRISMA Group. Preferred reporting items for systematic reviews and meta-analyses: the PRISMA statement. PLoS Med 2009;6: e1000097.

15 Hefti F, Müller W, Jakob RP, et al. Evaluation of knee ligament injuries with the IKDC form. Knee Surg Sports Traumatol Arthrosc 1993;1:226-34.

16 Wera JC, Nyland J, Ghazi C, et al. International knee documentation committee knee survey use after anterior cruciate ligament reconstruction: a 2005-2012 systematic review and world region comparison. Arthroscopy 2014;30:1505-12.

17 Jakob RP, Stäubli HU, Deland JT. Grading the pivot shift. Objective tests with implications for treatment. J Bone Joint Surg Br 1987;69:294-9.
18 Musahl V, Hoshino Y, Ahlden M, et al. The pivot shift: a global user guide. Knee Surg Sports Traumatol Arthrosc 2012;20:724-31.

19 Hedges LV, Vevea JL. Fixed-and random-effects models in meta-analysis. Psychol Methods 1998:3:486-504.

20 Higgins JP, Thompson SG, Deeks JJ, et al. Measuring inconsistency in metaanalyses. BMJ 2003;327:557-60.

21 Noyes FR, Barber-Westin SD. Revision anterior cruciate ligament surgery: experience from Cincinnati. Clin Orthop Relat Res 1996:325:116-29.

22 Johnson DL, Swenson TM, Irrgang JJ, et al. Revision anterior cruciate ligament surgery: experience from Pittsburgh. Clin Orthop Relat Res 1996;325:100-9.

23 Noyes FR, Barber-Westin SD. Revision anterior cruciate surgery with use of bone-patellar tendon-bone autogenous grafts. J Bone Joint Surg Am 2001;83:1131-43.

24 Taggart TF, Kumar A, Bickerstaff DR. Revision anterior cruciate ligament reconstruction: a midterm patient assessment. Knee 2004;11:29-36.

25 Carson EW, Anisko EM, Restrepo C, et al. Revision anterior cruciate ligament reconstruction: etiology of failures and clinical results. J Knee Surg 2004; 17:127-32

26 O'Neill DB. Revision arthroscopically assisted anterior cruciate ligament reconstruction with previously unharvested ipsilateral autografts. Am J Sports Med 2004;32:1833-41.

27 Ferretti A, Conteduca F, Monaco E, et al. Revision anterior cruciate ligament reconstruction with doubled semitendinosus and gracilis tendons and lateral extra-articular reconstruction. J Bone Joint Surg Am 2006;88:2373-9.

28 Garofalo R, Djahangiri A, Siegrist 0. Revision anterior cruciate ligament reconstruction with quadriceps tendon-patellar bone autograft. Arthroscopy 2006;22:205-14

29 Noyes FR, Barber-Westin SD. Anterior cruciate ligament revision reconstruction: results using a quadriceps tendon-patellar bone autograft. Am J Sports Med 2006:34:553-64.

30 Salmon LJ, Pinczewski LA, Russell VJ, et al. Revision anterior cruciate ligament reconstruction with hamstring tendon autograft: 5- to 9-year follow-up. Am J Sports Med 2006;34:1604-14.

31 Battaglia MJ, Ind, Cordasco FA, Hannafin JA, et al. Results of revision anterior cruciate ligament surgery. Am J Sports Med 2007;35:2057-66.

32 Denti M, Lo Vetere D, Bait C, et al. Revision anterior cruciate ligament reconstruction: causes of failure, surgical technique, and clinical results. Am J Sports Med 2008;36:1896-902

33 Diamantopoulos AP, Lorbach 0, Paessler HH. Anterior cruciate ligament revision reconstruction: results in 107 patients. Am J Sports Med 2008;36:851-60.

34 Wegrzyn J, Chouteau J, Philippot R, et al. Repeat revision of anterior cruciate ligament reconstruction: a retrospective review of management and outcome of 10 patients with an average 3-year follow-up. Am J Sports Med 2009;37:776-85

35 Muneta T, Hara K, Ju YJ, et al. Revision anterior cruciate ligament reconstruction by double-bundle technique using multi-strand semitendinosus tendon. Arthroscopy 2010;26:769-81.

36 Reinhardt KR, Hammoud $\mathrm{S}$, Bowers $\mathrm{AL}$, et al. Revision $\mathrm{ACL}$ reconstruction in skeletally mature athletes younger than 18years. Clin Orthop Relat Res 2012:470:835-42.

37 Franceschi F, Papalia R, Del Buono A, et al. Two-stage procedure in anterior cruciate ligament revision surgery: a five-year follow-up prospective study. Int Orthop 2013;37:1369-74.

38 Buda R, Ruffilli A, Di Caprio F, et al. Allograft salvage procedure in multiple-revision anterior cruciate ligament reconstruction. Am J Sports Med 2013;41:402-10.

39 Shelbourne KD, Benner RW, Gray T. Return to sports and subsequent injury rates after revision anterior cruciate ligament reconstruction with patellar tendon autograft. Am J Sports Med 2014;42:1395-400.

40 Kvist J, Ek A, Sporrstedt K, et al. Fear of re-injury: a hindrance for returning to sports after anterior cruciate ligament reconstruction. Knee Surg Sports Traumatol Arthrosc 2005;13:393-7.

41 Zaffagnini S, Russo RL, Marcheggiani Muccioli GM, et al. The Videoinsighte method: improving rehabilitation following anterior cruciate ligament reconstruction-a preliminary study. Knee Surg Sports Traumatol Arthrosc $2013 ; 21: 851-8$. 